\title{
Measuring, Modeling, and Predicting the Magnetic Assembly Rate of 2D Staggered Janus Particle Chains
}

\author{
Thomas W. Long*, Ubaldo M. Córdova-Figueroa ${ }^{+}$, and Ilona Kretzschmar ${ }^{*}$ \\ *Department of Chemical Engineering, City College of the City University of New York \\ (CUNY), New York, New York 10031, United States \\ ${ }^{+}$Department of Chemical Engineering, University of Puerto Rico - Mayagüez (UPRM), \\ Mayaguez, Puerto Rico 00681, United States
}

\section{Supporting Information}

The supporting information contains additional information regarding output obtained from our in-house tracking program such as particle traces, collision counts of assembly events involving singlets and other particle chains, evolution of chain concentrations as a function of time, and the cumulative probability function of nearest neighbors for all chains.

$\mathbf{F e}_{1-\mathrm{x}} \mathbf{O}$ JP System Assembly. Four videos of $2.4 \mu \mathrm{m} \mathrm{Fe} \mathrm{e}_{1-\mathrm{x}} \mathrm{O}$ Janus particles were taken for the analysis presented in the manuscript at $5 \mathrm{x}$ magnification for $700 \mathrm{~s}$ at 3 frames per seconds. Video S1 shows one of the four videos. The image frame shows a (768 by 960) $\mu \mathrm{m}^{2}$ area of the assembly cell with 1830 particles resulting in a particle area fraction of 1.1\%. The video has been modified from the original 10 min video taken at 3 frames per seconds to showing one frame every 10 seconds resulting in a 10x sped up video of 70 s length to fit the upload criteria and scales to $(480 \times 360)$ pixles². 


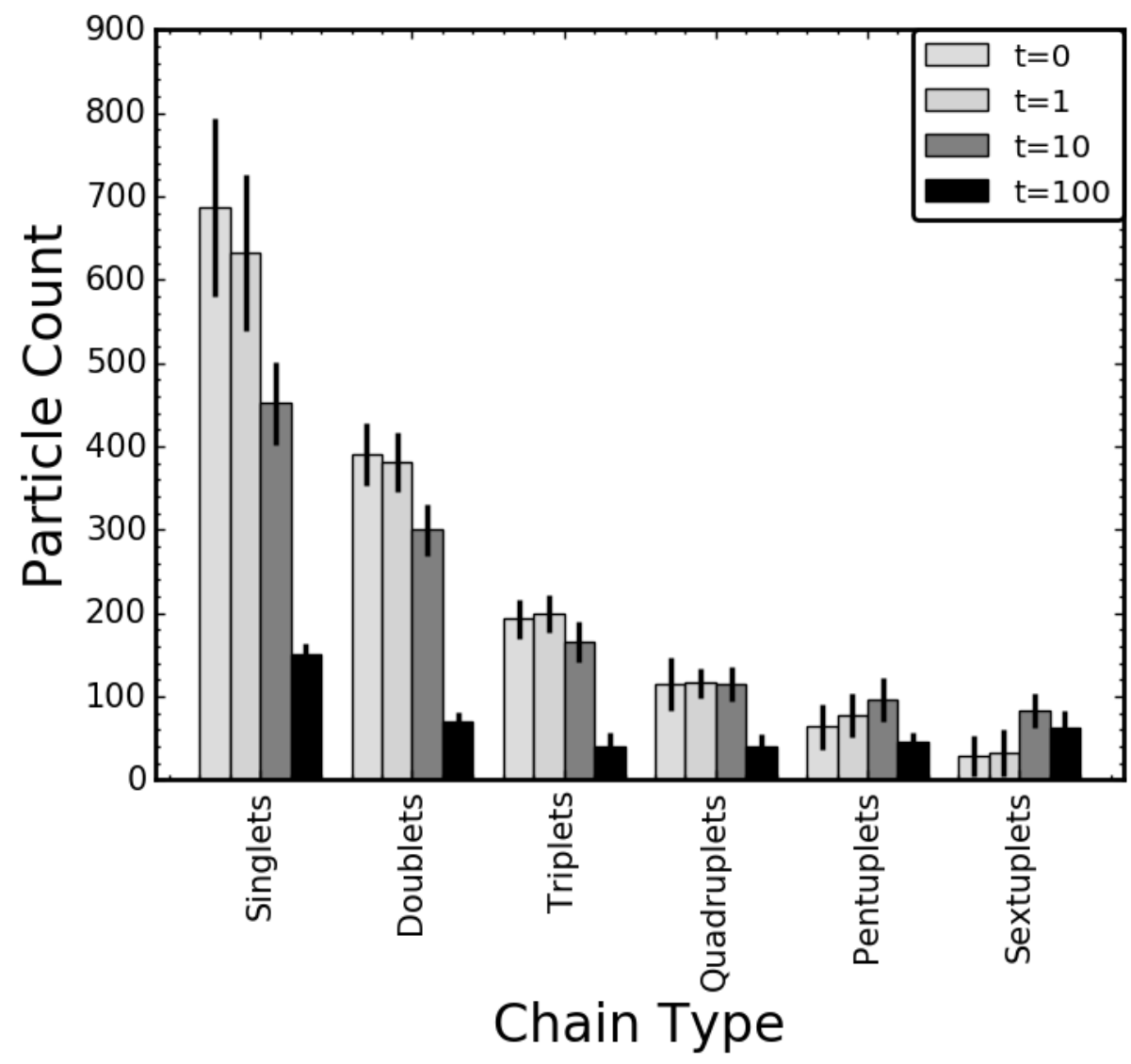

Figure S1: Total particle count contributing to chains of a particular length at four key times: $t=0,1,10$, and 100 obtained from averaging of four videos. Time is nondimensional $\left[\mathrm{t}^{*}=\mathrm{tD} / \mathrm{r}^{2}\right]$. Chain counts are weighted by the number of particles in the chain (i.e., each singlet adds 1 to the singlet count, each doublet adds 2 to the doublet count, etc.). Times for one type of chain are grouped to illustrate maxima in the occurrence of chains. Error bars indicate one standard deviation.

Chain Count as a function of experimental run time. Figure S1 shows histograms for chain counts from the four videos collected at time steps $t=0,1,10$, and 100, which represent experimental run times of $0,7,70$, and 700 s, respectively. Each bar shows the counts of a relevant particle chain (singlet, doublet, triplet, quartet, quintuplet, and sextuplet) at the time specified by the color of the bar with an error bar of one standard deviation. As expected, the number of singlet chains decreases and the number of chains with more particles increases as time goes on. Figure $\mathbf{S 1}$ demonstrates that the counts for chains with one or two particles decrease throughout the experiment, whereas counts for 
chains with three or more particles increase at short times and decrease at longer times going through a maximum value. At the maximum value the consumption events are occurring at a rate equal to formation events beyond which consumption of the chain dominates.

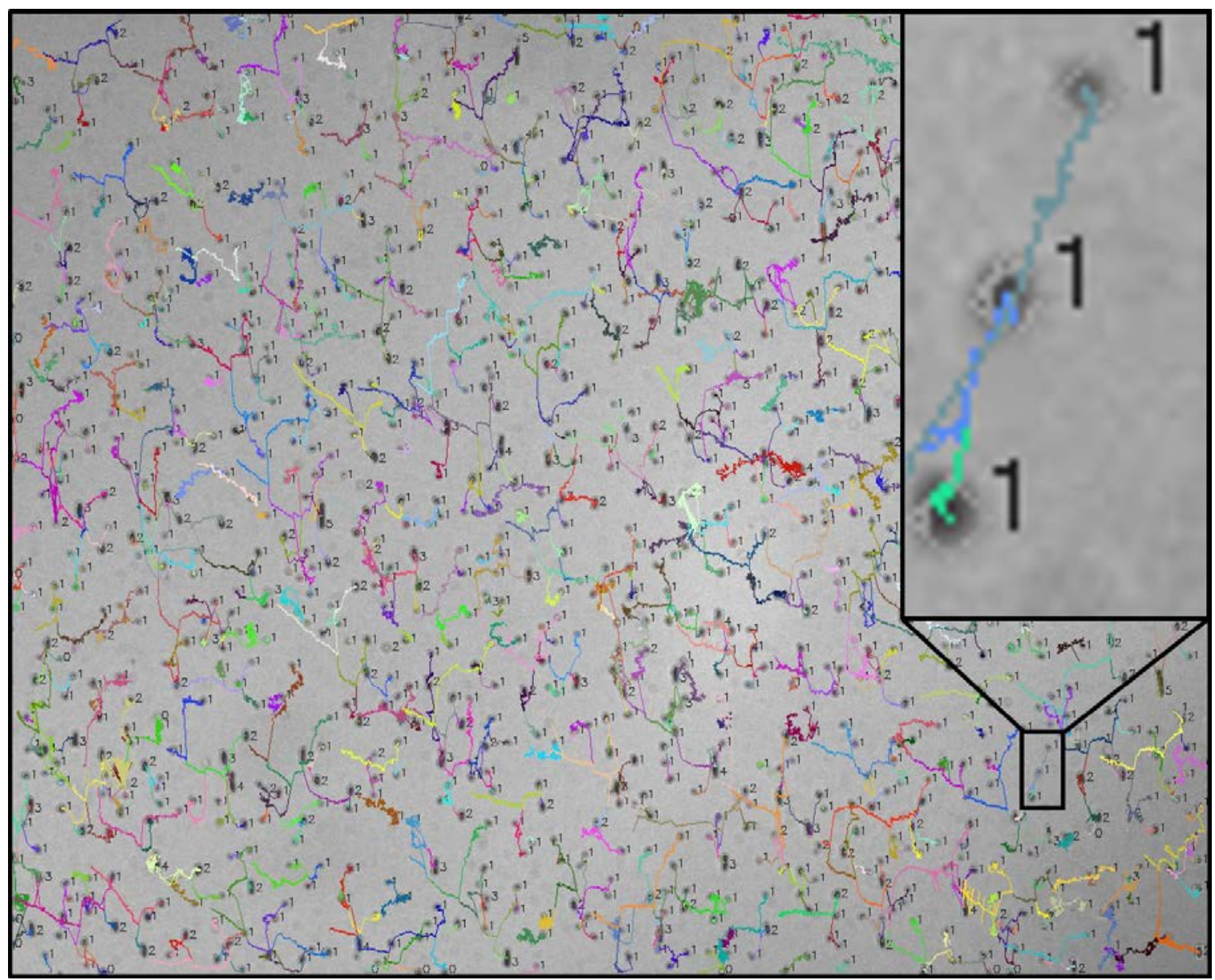

Figure S2: Video frame of $\mathrm{Fe}_{1-\mathrm{x}} \mathrm{O}$-capped JP system assembling in magnetic field of $B=0.08 \mathrm{~T}$ applied parallel to imaging plane at time $t=0$ with particle trajectories (colored lines) projected for $\Delta t=700 \mathrm{~s}$. Inset: Cropped snapshot of the section of the analyzed image to highlight chain collision events. Numbers displayed in the inset represent the number of particles in the chain as determined by contour at $t=0$. Intersecting trajectory lines indicate occurrence of an aggregation event.

Particle Tracking. Figure S2 shows the first frame $(t=0)$ from a video taken of $\mathrm{Fe}_{1-\mathrm{x}} \mathrm{O}-$ capped Janus particles assembling in a magnetic field of $B=0.08 \mathrm{~T}$ with particle trajectories (colored lines) projected for $\Delta t=700 \mathrm{~s}$ as obtained from our in house particle 
tracking analysis. The initial position and size of each chain is found through contour detection. The positions are tracked throughout the experiment and collision events are accounted for as the chains aggregate with each other. In the example shown in the inset of Figure S2, a singlet aggregates with a singlet. Thus, 2 is subtracted from the singlet count and 1 is added to doublet count. The resulting doublet then collides with another singlet. Now, 1 is then subtracted from both the singlet and doublet counts and 1 is added to the triplet count. Counting each event requires full trajectory information as opposed to the much faster method of independent frame analysis.

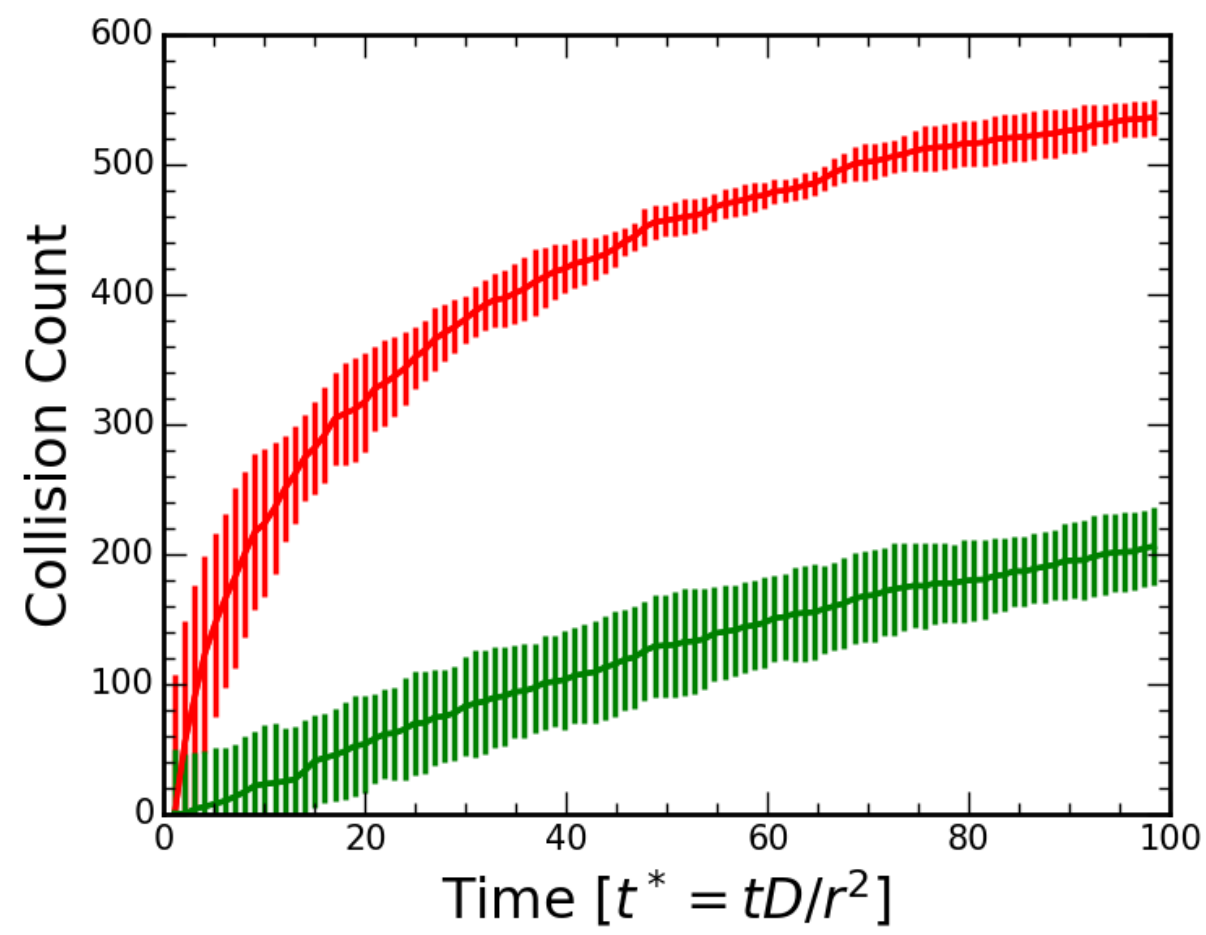

Figure S3: Count of collisions (i.e., aggregation events) in aggregating system averaged over four experimental videos. Red line represents count of all collision events involving singlets. Green line is a count of all other collision events. Error bars indicated one standard deviation.

Collision Event Counting. The Smoluchowski fitting introduced in the manuscript as Eq.

3a-3e operates under the assumption that aggregation events involving singlets are the 
major contributors to aggregation. Figure S3 demonstrates the validity of the assumption.

The cumulative sum of all aggregation events involving singlets averaged of four experiments is represented by the red line, whereas the cumulative sum of all collision events which do not involve singlets is shown in green. The percentage of collision events involving singlets at the end of the experiment is $70 \%$. The high percentage of events involving singlets justifies reduction of the degrees of freedom involved in the leastsquares fits based on Smoluchowski, i.e., Eq. 3a-3e, for the concentration profiles to only include events involving singlets.

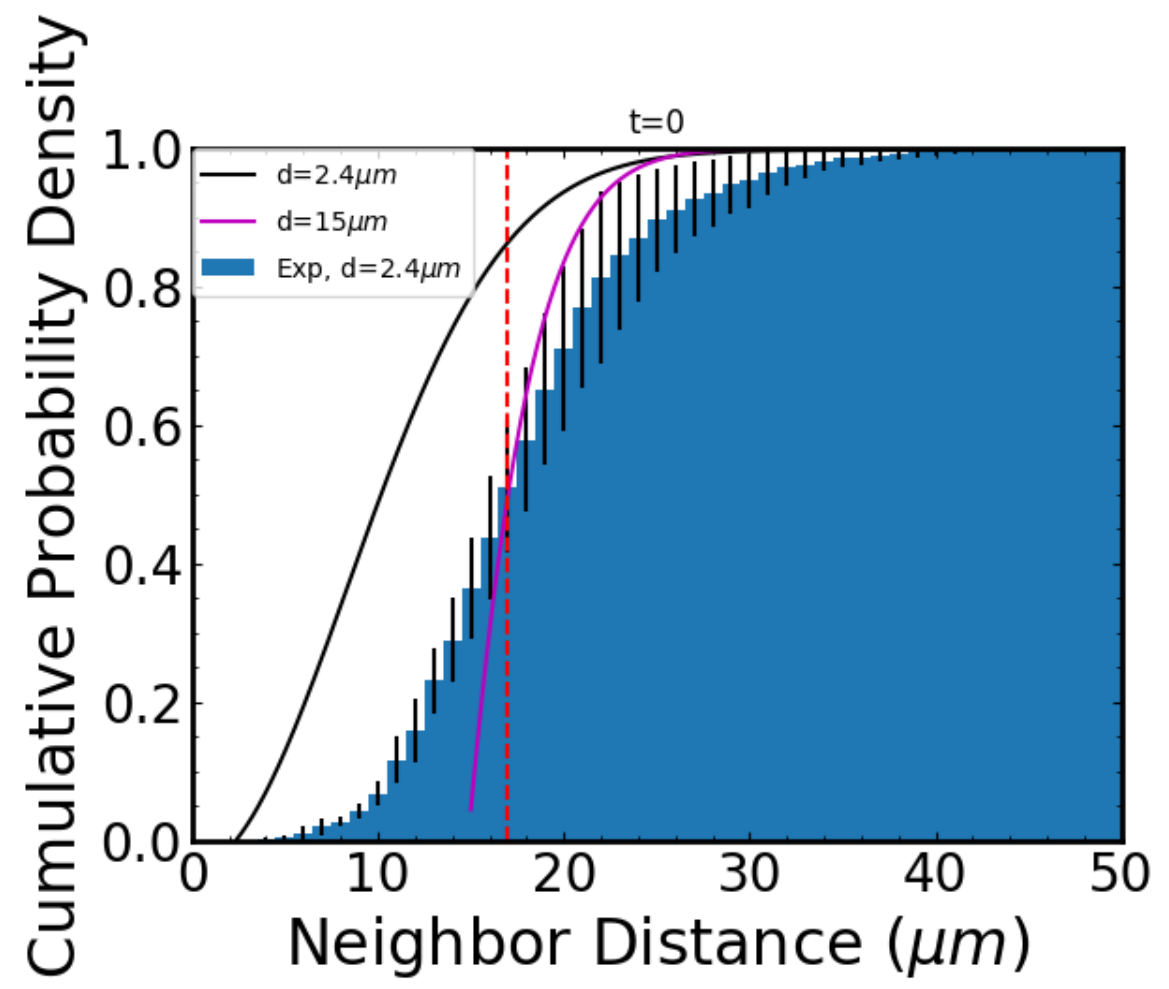

Figure S4: Cumulative probability function of nearest neighbors for all chains. The blue bars represent a histogram of the particle neighbor data obtained averaged from four videos. The black line is the nearest neighbor prediction for randomly distributed, non-interacting particles of the size used in our experiments ( $d$ $=2.4 \mu \mathrm{m}$ ). The red dashed line indicates the median obtained from the experimental nearest neighbor distance. The magenta line describes the nearest neighbor distribution obtained from a model that uses hardsphere particles with $d=15 \mu \mathrm{m}$ used to match the median nearest neighbor distance for the experimental 2.4 $\mu \mathrm{m} \mathrm{Fe}_{1-\mathrm{x}} \mathrm{O} \mathrm{JP}$ system. 
Capture Distance and Nearest Neighbor Distribution. Figure S4 depicts the particle neighbor data at $t=0$ for all chains as obtained from the average of four videos in a normalized histogram with error bars indicating one standard deviation. The data is collected by looping through each particle-particle pair and using conditional calculations to find the nearest neighbor for each particle. The list of neighbors is then binned as a histogram. The median nearest neighbor distance obtained for the $2.4 \mu \mathrm{m} \mathrm{Fe}_{1-\mathrm{x}} \mathrm{O}$ system is shown as a red dashed line.

The data obtained experimentally can be compared to models that predict the distribution and median nearest neighbor distance for randomly distributed systems. ${ }^{1}$ From comparison with the median nearest neighbor model (magenta line), it is apparent that the experimental $\mathrm{Fe}_{1-\mathrm{x}} \mathrm{O}$ Janus particle system is more closely aligned with a median nearest neighbor distance expected for a particle system with $d=15 \mu \mathrm{m}$ than $d=2.4 \mu \mathrm{m}$.

The capture distance model assumes that particles within the capture distance quickly aggregate with their respective cluster pair. The assumption is supported by the nearest neighbor analysis shown in Figure S4. Less than 15\% of the particles occupy the exclusion distance, e.g., distances less than the median nearest neighbor distance, that would be expected from a random particle distribution. A hard-sphere model with $d=15$ $\mu \mathrm{m}$ models the experimentally found median nearest neighbor distance (red dashed line) although it exhibits a steeper slope in the nearest neighbor distribution. The diameter of 15 $\mu \mathrm{m}$ compares favorably with both the average dipole-dipole least squares fit shown in Figure 4A (dashed line) and the capture distance, $r_{\text {cap }}$, of $16 \mu \mathrm{m}$ calculated using Eq. 22. The difference in the slopes of the experimental and predicted distributions is caused by particles spending a short amount of time within the capture distance while aggregating 
and some particles approaching each other but not aggregating. These events make the effective radius "softer" than what is expected from a pure hard sphere radius system.

The nearest neighbor analysis independently supports the capture radius model, indicating that the capture distance model describes a system of smaller particles as if it were made up of larger particles that immediately aggregate upon collision.

\section{Reference}

1. Torquato, S.; Lu, B.; Rubinstein, J., Nearest-neighbor distribution functions in many-body systems. Phys. Rev. A 1990, 41 (4), 2059-2075. 